\title{
Nivel de desarrollo de las nociones de seriación y clasificación de los estudiantes, Amazonas, Perú
}

\section{Level of development of the notions of seriation and classification of students, Amazonas, Peru}

\author{
Irwen Segura Cotrina ${ }^{1}$, Jimena García Trinidad ${ }^{2}$, José Darwin Farje Escobedo ${ }^{3}$
}

\section{RESUMEN}

El presente trabajo tuvo como objetivo principal diagnosticar el nivel de desarrollo de las nociones de seriación y clasificación de los estudiantes del primer grado de la institución educativa 18121 de la localidad de Guadalupe, Amazonas, 2020; la investigación fue de tipo básica, de nivel descriptivo con diseño descriptivo simple; La muestra estuvo conformada por 20 estudiantes. El instrumento utilizado para la recolección de datos fue una ficha de observación de escala tipo Likert, con 10 ítems para cada noción, con los niveles de deficiente, regular y eficiente. Según los resultados, en la noción de seriación, el 10\% se encuentra en el nivel deficiente, y el 40\% está en el nivel regular; mientras que en la noción de clasificación, el 35\% está en el nivel regular y el 60\% alcanza el nivel eficiente; comparando el nivel de logro de ambas dimensiones. Se concluye que los alumnos presentan dificultades para resolver ejercicios de seriación y clasificación, utilizando material concreto, con una ligera ventaja en las actividades relacionadas a clasificar objetos; lo que es un indicador que los estudiantes aún no logran los aprendizajes esperados para el grado.

Palabras clave: Noción de seriación, noción de clasificación

\begin{abstract}
The main objective of the present work was to diagnose the level of development of the notions of seriation and classification of first grade students of the educational institution 18121 in the town of Guadalupe, Amazonas, 2020; The research was of a basic type, descriptive level with a simple descriptive design; The sample consisted of 20 students. The instrument used for data collection was a Likert-type scale observation sheet, with 10 items for each notion, with the levels of deficient, regular and efficient. According to the results, in the notion of seriation, $10 \%$ are at the deficient level, and $40 \%$ are at the regular level; while in the notion of classification, $35 \%$ are at the regular level and 60\% reach the efficient level; Comparing the level of achievement of both dimensions, it can be seen that the students have difficulties in solving serialization and classification exercises, using concrete material, with a slight advantage in the activities related to classifying objects; which is an indicator that the students have not yet achieved the expected learning for the grade.
\end{abstract}

Keywords: Notion of seriation, notion of classification

\footnotetext{
${ }^{1}$ Bachiller en Educación de la Facultad de Educación y Ciencias de la Comunicación de la Universidad Nacional Toribio Rodríguez de Mendoza de Amazonas.

${ }^{2}$ Bachiller en Educación de la Facultad de Educación y Ciencias de la Comunicación de la Universidad Nacional Toribio Rodríguez de Mendoza de Amazonas.

${ }^{3}$ Docente Asociado, de la Facultad de Educación y Ciencias de la Comunicación de la Universidad Nacional Toribio Rodríguez de Mendoza de Amazonas.
} 


\section{INTRODUCCIÓN}

En el mundo actual, la educación resulta ser, de todas maneras, el pilar del desarrollo en cualquier contexto social, y son las escuelas espacios formativos que deben responder al encargo social; sin embargo, el trabajo educativo cada vez un reto de mayor complejidad, porque tiene que enfrentar a una serie de barreras, obstáculos y brechas que se van posicionando más. Pero los países hacen esfuerzos mancomunados orientados a la mejora de la educación. Según el Banco Mundial (2017) "en los países que se consideran en vías de desarrollo, se ha incrementado de dos a siete años la media en el tiempo de estudio, durante el período 1950 y 2010; sumado a esto, cada vez va incrementándose la tasa de estudiantes que no dominan las competencias elementales y básicas que constituirán cualidades para desenvolverse en la cotidianeidad, con solvencia".

La Matemática, la Comunicación y Personal Social, son áreas básicas dentro del currículo, pero lamentablemente en nuestro país, los estudiantes muestran mayores dificultades, sobre todo en matemática, a pesar que debe ser abordado como parte de la cotidianeidad y que toda persona convive con ella de manera personal y social, habitualmente para satisfacer necesidades y solucionar problemas; sin embargo, los estudiantes de básica y superior, en su mayoría lo abordan con pánico; al respecto el Ministerio de educación (2018), muestra los resultados de la Evaluación Censal de Estudiantes ECE, donde se aprecia que un $9,3 \%$ se encuentra en el nivel "previo al inicio"; el 19,3\%, en el nivel "inicio"; un 40,7\% en el nivel de "proceso"; es decir, cerca al $70 \%$ de los estudiantes del cuarto grado de educación primaria, no logra desarrollar capacidades matemáticas.

La precaria calidad de nuestra educación básica, se va ver más afectada por la presencia del coronavirus Covid-19, que ha obligado al gobierno declarar el estado de emergencia nacional y ha dispuesto el aislamiento social obligatorio (Presidencia del Consejo de Ministros, 2020); lo que hace sospechar, según la Defensoría del Pueblo (2020) que los niños tendrán mayores dificultades para aprender en sus hogares a través de la estrategia "Aprendo en casa", debido a las grandes brechas digitales existentes en nuestro país y también a la escasa cultura de apoyo de los padres de familia en las responsabilidades educativas.

Los escolares de la región Amazonas, también tienen dificultades para aprender significativamente el área de Matemática, situación que se incrementa aún más si se tiene en cuenta la brecha entre el servicio educativo rural y urbano, tal como los muestran los resultados del reporte de la prueba de evaluación censal de estudiantes, correspondientes a nuestro departamento (Minedu, 2018).
Situación similar ocurre en el distrito de Camporredondo, particularmente en el anexo de Guadalupe, ubicada en la zona rural, donde los estudiantes de educación primaria presentan dificultades en el desarrollo de capacidades asociadas al área de Matemática, particularmente sobre las nociones de seriación y clasificación.

La presente investigación se justifica por cuanto estudia un tema de interés en el escenario educativo y que es abordado de manera empírica por los docentes de educación primaria y de otros niveles educativos, nos referimos a las nociones de seriación y clasificación, que se trabajan de manera superficial con los niños y niñas; aun cuando Gómez (2017), refiere que "las actividades orientadas al desarrollo de nociones prenuméricas, forman parte de la vida práctica de los niños, que permiten fortalecer sus habilidades y destrezas para el aprendizaje de número, además, estimulan los sentidos al estar en contacto con material concreto"; en ese sentido, está presente la sustentación o fundamentación teórica, que se basa entre otros, en el método Montessori.

En la dimensión práctica, el estudio se justifica porque se ocupa de una problemática que está presenta en el escenario real, donde los escolares presentan dificultades para la iniciación en la matemática en el nivel primario y al no ser abordados de manera oportuna y adecuada; los niños van avanzando en su escolaridad y paralelamente se va acrecentando la fobia y el temor por aprender matemática, cuyos resultados se ven reflejados en las pruebas de evaluación censal de estudiantes.

Además, se justifica metodológicamente porque en todo el proceso investigativo se han utilizado diferentes métodos, técnicas e instrumentos y procedimientos, de manera sistemática para darle consistencia científica al estudio, de tal manera que los resultados obtenidos constituyan la base para otras investigaciones relacionadas la tema en particular.

El estudio tiene como objetivo general diagnosticar el nivel de desarrollo de las nociones de seriación y clasificación de los estudiantes del $1^{\circ}$ grado de la institución educativa 18121 de Guadalupe, Amazonas, 2020. Y en base a los objetivos específicos, la investigación persigue: a) medir el nivel de desarrollo de la noción de seriación de los estudiantes que participaron en la investigación; b) evaluar el nivel de desarrollo de la noción de clasificación de los estudiantes que formaron parte de la muestra; y, c) comparar el nivel de desarrollo de las nociones de seriación, clasificación de estudiantes de $1^{\circ}$ grado.

Asimismo, se han considerado los antecedentes de estudio que a continuación se indica: Priego (2018), en su investigación sobre clasificación, seriación y correspondencia término a término: un estudio en un 
aula de educación escolar. El estudio fue exploratorio y se desarrolló con seis alumnos; la técnica que utilizó es la observación, registrando sus resultados en una ficha de observación; las conclusiones finales fueron: a) los niños y las niñas se desenvuelven espontáneamente desarrollando actividades con materiales manipulativos, contribuyendo así a fortalecer la base del razonamiento lógico matemático, b) para fomentar la lógica es conveniente diseñar y desarrollar distintas actividades utilizando materiales manipulativos, con los que puedan experimentar y de esta forma facilitarles el aprendizaje.

Atencia (2017), en su estudio sobre Nociones básicas para la construcción del número: clasificación y seriación de niños de cinco años de la institución educativa 377 de Los Olivos; su diseño fue no experimental, de corte transversal; la muestra estuvo conformada por 95 niños/as matriculados en el aula de cinco años; como instrumento utilizó una escala de estimación, las conclusiones finales fueron: a) en la muestra de estudio, las noción básica de seriación, no están bien desarrolladas, se encuentran en proceso con un $92,6 \%$, ello significa que no demuestran solvencia en el manejo de esas nociones, esperadas para su edad; probablemente sea por el limitado uso de material concreto, o la falta de apoyo en la casa como también las constantes inasistencias a su institución; b) en la noción de clasificación, el 68,4\% de los niños, están en proceso, es decir se encuentran en camino de lograr agrupar objetos en función a criterios diversos.

Balcázar (2017), en su estudio sobre Un programa de juegos didácticos para mejorar la clasificación y seriación en niños de primer grado de la institución educativa primaria de colegios y academias Montessori Chiclayo; su diseño fue de tipo explicativo en nivel cuantitativo, en nivel preexperimental, con diseño preprueba y posprueba con un solo grupo; la muestra estuvo conformada por 20 alumnos, de los cuales 8 fueron varones y 12 mujeres, matriculados en el primer grado, el instrumento utilizado fue la lista de cotejo; las conclusiones finales alcanzadas son: a) La aplicación del pretest identificó un nivel bajo en las nociones de clasificación obtenido, con "39" en el "si”" y con "61" en el "no"; siendo muy parecido el resultado en la noción de seriación; sin embargo, el desarrollo del programa notó una gran mejoría en los niños notando el alto nivel en el desarrollo de ambas nociones, superando en promedio el $72 \%$ de efectividad; b) el programa pedagógico didáctico resultó ser muy trascendente para superar las dificultades que presentaban los niños en el desarrollo de las dos nociones básicas, pues les permitió mejorar de manera importante su desempeño.

Correa (2019), en su investigación sobre la Influencia de los juegos tradicionales para mejorar el aprendizaje de las operaciones matemáticas de suma y resta en estudiantes de la institución educativa $\mathrm{N}^{\circ}$ 18255, Leoncio Prado de Chachapoyas. Su objetivo principal fue, mejorar el aprendizaje de las operaciones matemáticas en estudiantes de primer grado de la institución educativa Leoncio Prado, a través de actividades lúdicas. Como instrumento de recolección de datos utilizó un pretest y un postest; trabajó con una muestra de 15 estudiantes, arribando entre otras a la siguiente conclusión: a) La vida de los niños es jugar y juegan por instinto, por esa fuerza interna que los obliga a moverse. En nuestra investigación, los aprendizajes, en promedio general en el pretest fue de 6.83 y por efecto del desarrollo de la estrategia, el promedio en el postest fue de 15.37, con un avance bastante importante.

El trabajo se sustenta en las bases teóricas descritas a continuación: Desarrollo del pensamiento de la matemática, Vigotsky y Piaget (citado por Varas y Zarco, 2017), al referirse al desarrollo del pensamiento de la matemática, sostienen:

Que, en el desarrollo del pensamiento, están presentes dos ciencias que explican el proceso que está asociado al aprendizaje de los niños/as, como son por un lado, la psicología cognitiva y por otro, la neurociencia, que, sincronizando con la primera disciplina, contribuyen a la comprensión de la forma de adquisición del conocimiento; además, también la forma de aprendizaje por parte del niño al estar en contacto con su entorno; en el segundo caso, el desarrollo del cerebro permite vincular procesos del pensamiento con las habilidades del lenguaje, la motricidad, y en general la activación de los sentidos. En esa dirección, las teorías: sociocultural de Vigotsky y de la construcción del conocimiento de Piaget, fundamentan el desarrollo del pensamiento matemático en el sentido que influye directamente en la enseñanza aprendizaje. Asimismo, Piaget distingue tres tipos de conocimiento a ser adquiridos por el individuo: físico, lógico-matemático y social; el primero físico, es el que rodea a la persona y lo conforman los objetos del mundo natural, con sus diferentes propiedades (dureza, rigurosidad, peso, sabor, textura); éstas son apropiadas por el infante a través de la manipulación de los objetos cercanos al niño. A través de la observación el niño abstrae, la forma, el color, tamaño, y también otras propiedades del objeto. Las propuestas planteadas por los científicos precitados, también son fortalecidos por Montessori (como se citó en Sanchidrián (2017), cuando sostiene que los niños enriquecen y nutren sus experiencias matemáticas cuando se relacionan con materiales y utensilios diversos y los asimilan con los sentidos (pág. 37).

La enseñanza de la matemática, básicamente a inicios de la escolaridad, no solo es una cuestión asociada directamente a la didáctica, va mucho más allá; involucra al proceso de desarrollo cognitivo del niño, la organización de la clase, y de manera puntual, también tiene que ver con la intervención del docente, 
los recursos y/o materiales que se utilizan, entre otros; sumado a esto, aspectos relacionados a la armonía que debe haber entre el niño y el contexto escolar, que desde hace buen tiempo, lo viene pensando, antes de participar en su primer día de clase; al respecto, Panizza (1999), sostiene que es "importante que el alumno brinde sentido y significancia a lo que está haciendo en matemática; cómo lograr que encuentren significado a la actividad matemática para superar la mecanización; hecho de proveer sentido al acto de matematización, debe ser preocupación e interés cotidiano de docentes enseñantes de la matemática escolar."

Las teorías asociadas al conocimiento matemático, principalmente son: la conductista, considera que los niños llegan a la escuela como recipientes vacíos los cuales hay que ir llenando; solamente traen en sus conocimientos previos, algunas técnica de conteo mecánico o automatizado, de memoria; que en términos de significancia o andamiaje para iniciarse en la matemática, muy poco contribuye; $\mathrm{y}$, también está la teoría cognitiva, considera que, antes de empezar sus primeros estudios escolares, los niños han adquirido todo un amplio repertorio de conocimientos matemáticos; que está asociados a agrupar, manipular, asociar, realizar conteos, construir a partir de materiales que le rodean.

Las nociones básicas en la matemática. El pensamiento lógico matemático, concebido como la facultad de formar, construir y relacionar ideas o sucesos y también eventos, mediante proceso cognitivos; siguiendo reglas formales de deducción y cuyas conclusiones se van gestando a partir de una serie de premisas válidas y confiables. Asimismo, el pensamiento lógico matemático tiene su base en las vivencias y experiencias, pero también a la interacción que protagoniza el niño con su mundo inmediato; ello le permite al niño caracterizar y establecer relaciones entre objetos, explorar materiales, establecer semejanzas y diferencias, y otras actividades más.

Según Piaget (como se citó en Castro, 1992), "la construcción de número es correlativo con el desarrollo del pensamiento lógico, y que al nivel prelógico se corresponde con un periodo prenumérico", además agrega que el conocimiento numérico no viene dado, tampoco se adquiere súbitamente, sino que se llega a través de un camino que va evolucionando desde la infancia hasta la madurez.

Las nociones básicas, se clasifican en noción espacio temporales (está asociado al manejo del espacio a partir de la actividad con su propio cuerpo); noción de comparación (se asocia a "par", equiparar, comparar de acuerdo a criterios diversos); noción de clase o clasificar (agrupar objetos en base a criterios); y la noción de seriación (ordenamiento sistemático de las diferencias entre los elementos que corresponden a un mismo grupo).
Por otro lado, el sentido numérico, está dirigido a un conjunto flexible de capacidades y habilidades de orden cognitivo, en el cual está inmerso el cálculo mental, las estimaciones numéricas, elaboración de una serie de cuantificadores, en consecuencia se puede decir que una persona se empodera de un sentido numérico cuando hace gala de una buena intuición sobre los números así como las relaciones existente entre ellos, por lo que, se dice que ese sentido numérico constituye un aspecto especial que exige pensar a los niños, por ahí va la importancia de su desarrollo desde edades tempranas para que en adelante demuestren competencias y habilidades, así como, pierden ese temor por hacer de la matemática un instrumento que les permita actuar en la vida cotidiana (Castro, Rico y Castro, 1995).

La preparación para el aprendizaje de la matemática, se gesta en el nivel preescolar, pero también durante los dos primeros grado de educación primaria; y está relacionada básicamente al desarrollo de nociones básicas de seriación, clasificación, conservación de cantidad, entre otras; sin embargo, en la realidad concreta se percibe que el docente privilegia otros contenidos temáticos que son ajenos a los intereses de los alumnos; generando una brecha en la construcción de bases sólidas para posteriores aprendizajes en esta área; por lo que, se pretende conocer el nivel de desarrollo de las nociones básicas de seriación y clasificación en estudiantes que están cursan el primer grado de educación primaria. Es por esta razón que las orientaciones deben direccionarse de tal manera que los docentes de educación preescolar y escolar, sean capaces de incluir técnicas, , formas, modos que estén relacionados a experiencias enriquecedoras a partir de la relación con material concreto y en situaciones significativas, en esas circunstancias, los profesores cumplen un rol fundamental como orientadores y monitores.

\section{Clasificación}

La clasificación está asociada a la ejecución de acciones que permiten, entre otras tareas, la formación de conjuntos o subconjuntos, enfatizando en la propiedad de agrupación por la forma, tamaño; también por el color, espesor, entre otros. Dicho de otro modo, Como se menciona, es la unión de objetos que suponen relación de semejanza entre elementos que conservan similitud, que pone en juego para desarrollar nociones matemáticas.

La habilidad de clasificar tiene una particularidad en los niños, está asociada al establecimiento de semejanzas y diferencias entre los elementos que le resultan interesante, llegando a formar sub clases, de modo que, luego lo incluirá en una clase de mayor extensión.

Según Rencoret (1995), la clasificación, constituye una actividad propiamente de los humanos, y en definitiva, consiste en ordenar diversos elementos $u$ objetos teniendo presente criterios comunes. Por su parte Gutiérrez (2011) dice que "es la destreza que 
permite al educando concretar acciones que se asocian a distribuir, ordenar, dividir por clases o categorías, es decir, determinar la clase o grupo al que corresponde un elemento."

Es sumamente importante desarrollar la noción de clasificación, desde la primera infancia, dado que permite aprender a ser lógico (Nunes y Bryant, 2005), en ese sentido el niño que reconozca reglas lógicas, de todas maneras, puede entender y realizar adecuadamente una variedad de tareas matemáticas. En ese contexto, es necesario que se propicien y construyan tres operaciones lógicas sustanciales: la clasificación, la seriación y la correspondencia; y se van construyendo de modo simultáneo y no en forma sucesiva; que en adelante conducirán al niño a concebir progresivamente el concepto de número, a partir de las experiencias directas como resultado de estar en contacto con materiales y recursos.

Con referencia a lo anterior la clasificación, es una de las nociones sustanciales y el vehículo que lleva al niño al concepto de número, por lo que, resulta de suma relevancia, ofrecerle desde la etapa preescolar retos numéricos, acorde a su edad; donde utilizando sus sentidos, irán explorando, manipulando, indagando, construyendo, armando y realizando otras acciones relacionadas a la naturaleza del material, y en ese afán de interactuar, van aprendiendo y preparándose para los aprendizajes posteriores relacionados con número y numeración.

\section{Seriación}

Habilidad que conduce a efectuar comparaciones, establecer relaciones asimétricas, lo que implica desarrollar las nociones de orden y series, utilizando material concreto.

La seriación, es la habilidad mediante el cual, el infante demuestra experticia para ordenar objetos de acuerdo a una característica, consigna, criterio o dimensión dada, estableciendo relaciones entre ellos, en función a sus cualidades, particularidades o características. Pero también, como refiere la Guía Tutorial del Ministerio de Educación (2011), pueden establecer relaciones de asimetría en un conjunto de objetos; esta acción se va convirtiendo en operacional dado que se pone en juego el principio de reversibilidad.

Pero también es necesario precisar que en la seriación, de todas maneras, existe el criterio de transitividad, habilidad lógica del pensamiento que está asociada a relacionar elementos u objetos, en un mismo sentido, ya sea de manera creciente o decreciente, a partir de la naturaleza del material utilizado.

Los niños en edad prescolar y primeros años de su escolaridad, en su mayoría presentan dificultades para resolver ejercicios o actividades relacionadas a la seriación, cuando tienen entre cuatro y seis años, van incluyendo en su juego libre algunas acciones que constituyen señales de la noción de seriación, cuando ordenan objetos en función a criterios específicos, pero todas esas actividades forman parte de su juego libre, es allí donde el acompañante, monitor o docente debe estar presente para presentar nuevas consignas a los niños (Vera, 2009).

Para Condemarín, et al (1986), es una operación relacionada al ordenamiento en base a variaciones de una o más características y que involucra los conceptos siguientes: i) transitividad, permite construir la seriación a partir de la comparación de tres elementos, del modo siguiente: objeto $\mathrm{A}$, más pequeño que objeto $\mathrm{B}$, y objeto $\mathrm{B}$ más pequeño que objeto $\mathrm{C}$; entonces objeto $\mathrm{A}$ es más pequeño que el objeto C. ii) reversibilidad, se refiere a la movilización del pensamiento en dos direcciones inversas, en base al ejemplo anterior, se puede decir: A es más pequeño que $C$, pero también $C$ es más grande que $\mathrm{A}$.

\section{MATERIAL Y MÉTODO}

La investigación fue de tipo básica, de nivel descriptivo.(Hernández, Fernández y Baptista, 2010).

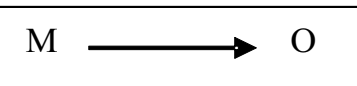

Donde:

\section{M : $\quad$ Muestra \\ $\mathrm{O}$ : $\quad$ Observación de la muestra}

La población y muestra lo conformaron 20 alumnos de la institución educativa primaria $\mathrm{N}^{\circ} 18121$ de la localidad de Guadalupe, Amazonas. El muestreo fue no probabilístico, por conveniencia.

El instrumento fue una ficha de observación, conformado por 10 ítems para la dimensión de seriación y 10 ítems para la dimensión de clasificación; con escalas valorativas de deficiente, regular y eficiente, cada uno con sus respectivas puntuaciones.

\section{RESULTADOS}

Tabla 1

Nivel de desarrollo de la noción de seriación de alumnos de educación primaria

\begin{tabular}{|l|ccc|}
\hline \multicolumn{4}{|c|}{ Dimensión: seriación } \\
\hline Niveles & Frecuencia & Porcentaje & P. acumulado \\
Deficiente & 2 & 10.0 & 10.0 \\
Regular & 8 & 40.0 & 50.0 \\
Eficiente & 10 & 50.0 & 100.0 \\
Total & 20 & 100.0 & \\
\hline
\end{tabular}


Los resultados generan preocupación, dado que, en el $1^{\circ}$ grado de educación primaria, los docentes estilan iniciar la enseñanza de la numeración, aun cuando los niños no desarrollan a plenitud una noción básica para la iniciación de número. Se puede observar que en el nivel de desarrollo de la noción de seriación, donde el $50 \%$ de los estudiantes están en el nivel eficiente y el otro $50 \%$ se encuentra en los niveles de eficiente y regular.

\section{Tabla 2}

Nivel de desarrollo de la noción de clasificación de los alumnos de educación primaria

\begin{tabular}{|l|ccc|}
\hline \multicolumn{4}{|c|}{ Dimensión: Noción de clasificación } \\
\hline Niveles & Frecuencia & Porcentaje & P. acumulado \\
Deficiente & 1 & 5.0 & 5.0 \\
Regular & 7 & 35.0 & 40.0 \\
Eficiente & 12 & 60.0 & 100.0 \\
Total & 20 & 100.0 & \\
\hline
\end{tabular}

En lo que respecta al nivel de desarrollo de la noción de clasificación, los resultados tampoco son halagadores, dado que el $40 \%$ de los estudiantes se ubican en los niveles de deficiente y regular en el dominio de la habilidad de clasificar objetos; sin embargo, ya están desarrollando contenidos relacionados a la numeración.

\section{Figura 1}

Resultado comparativo del desarrollo de la noción de seriación y de clasificación de los alumnos de primer grado de educación primaria.

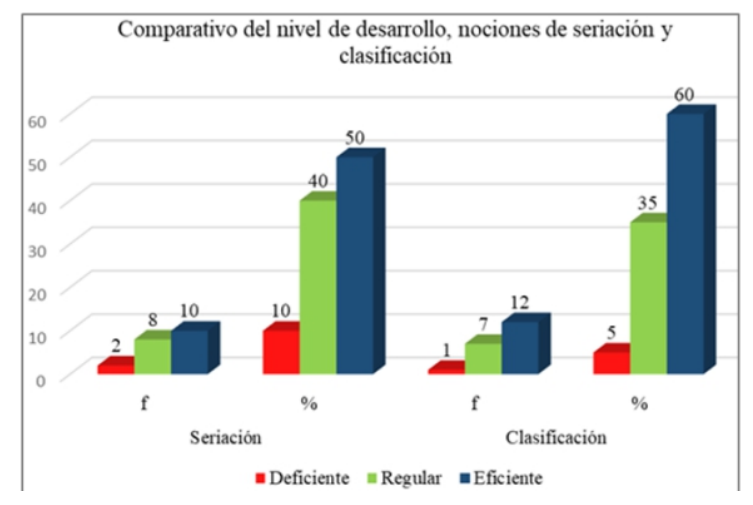

Haciendo el análisis comparativo de los resultados, se aprecia que, existe una ligera mejora en la noción de clasificación; sin embargo, el problema está presente en el aula, dado que un buen grupo de estudiantes no dominan a plenitud resolver ejercicios relacionados a nociones básicas que son prerrequisitos para la iniciación en número $\mathrm{y}$ numeración.

\section{DISCUSIÓN}

A partir de los hallazgos encontrados, acerca del nivel de desarrollo de las nociones de seriación y clasificación en los estudiantes del primer grado de la institución educativa $\mathrm{N}^{\circ} 18121$ de Guadalupe, se aprecia que en promedio, casi la mitad de los escolares presentan dificultades para resolver ejercicios de clasificación con material concreto, estableciendo semejanzas o diferentes entre objetos, al respecto, Boule (1995), refiere que los niños, citando a Piaget, refiere que la habilidad de clasificar se inicia en el periodo preoperacional, es decir entre los 2 y 7 años; en consecuencia los docentes deben desarrollar actividades que contribuyan a la consolidación de la noción de clasificación teniendo en cuenta diferentes criterios.

Según los resultados, se aprecia que en la noción de seriación, una cantidad significativa de estudiantes que participaron en el estudio, el 50\% se encuentran en los niveles de deficiente y regular, es decir presentan algunas dificultades cuando se les pide que establezcan seriaciones con material concreto; encontrando similitudes con el estudio de Atencia (2017), en su estudio sobre nociones básicas para la construcción de número: clasificación y seriación, concluye que, los alumnos no tienen bien desarrollada la noción de seriación, dado que el 92\% se encuentra en proceso; lo que significa que carecen de solvencia para desarrollar ejercicios de seriar objetos utilizando material concreto.

En la noción de clasificación, se aprecia que el 35\% de estudiantes del primer grado de educación primaria se encuentran en el nivel medio y el $60 \%$ es decir más de la mitad logra un desempeño eficiente; comparando con el estudio de Balcázar (2017), sobre programa de juegos didácticos para mejorar la clasificación seriación en niños de primer grado de colegios y académicas Montessori de Chiclayo; donde concluye que en el pretest, los estudiantes se encuentran en un nivel bajo, dado que el $61 \%$ no logran resolver ejercicios relacionados a clasificar objetos de acuerdo a criterios diferenciados.

Finalmente, cuando comparamos los resultados obtenidos, en el logro de las nociones de seriación y clasificación, se aprecia que una parte importante de la muestra de estudio presentan dificultades, presentando una ligera ventaja en la noción de clasificación; aun cuando el material didáctico utilizado forma parte de los kits educativos conocidos para los niños, como son los bloques lógicos, las regletas de Cuisenaire, entre otros, probablemente la dificultad esté asociado a que los docentes utilizan muy poco el material concreto, sumado a esto, niños evaluados estudian en una institución educativa rural. 


\section{CONCLUSIONES}

El nivel de desarrollo de los estudiantes del primer grado de educación primaria de la institución educativa primaria 18121 de la localidad de Guadalupe, en la noción de seriación, de una muestra de 20 estudiantes, el 50\% alcanzan el nivel de eficiente, el 10\%, el nivel deficiente y el $40 \%$ está en el nivel regular.

Los estudiantes que participaron en el estudio, en el nivel de desarrollo de la noción de clasificación, muestran que el 60\% alcanzan el nivel eficiente, el $5 \%$, el deficiente y el $35 \%$, el nivel regular, encontrando una ventaja con respecto a la noción de seriación.

Cuando se hace la comparación en el nivel de desarrollo de las nociones de seriación y clasificación, los estudiantes en ambos casos presentan dificultades para resolver las consignas dadas; situación que constituye una problemática dado que los docentes descuidan el logro de las nociones prenuméricas básicas como prerrequisito para el abordaje de la enseñanza de la numeración

\section{REFERENCIAS BIBLIOGRÁFICAS}

Atencia, G. (2017). Nociones básicas para la construcción del número: clasificación y seriación de niños de cinco años de la institución educativa 377 "Divino Niño Jesús, Los Olivos, (tesis de pregrado). Universidad César Vallejo.

Balcázar, G. (2017). Programa de juegos didácticos para mejorar la clasificación y seriación en niños y niñas de primer grado de la institución educativa primaria de colegios y academias Montessori Chiclayo, (tesis de pregrado). Universidad Católica Los Ángeles de Chimbote.

Boule, F. (1995). Manipular, organizar y representar: Iniciación a las matemáticas. Madrid: Narcea.

Castro, E. (1992). Números y operaciones fundamentos para una aritmética escolar. Síntesis.

Castro E, Rico L, Castro E, (1995). Estructuras aritméticas elementales y su modelización. Grupo editorial Iberoamericana.

Condemarín, M., Chadwick, M. y Milicic, N. (1986). Madurez escolar. Andrés Bello.

Correa, R. (2019). Influencia de los juegos tradiion ales para mejorar el aprendizaje de las operaciones matemáticas de suma y resta en los estudiantes de la institución educativa $N^{\circ}$ 18255, Leoncio Prado, Chachapoyas, 2018. (Tesis de pregrado). UNTRM.

Cultura (1 de octubre de 2019). La Educación escolar está en crisis a nivel global. Informe del Banco Mundial. https://mundo. sputniknews.com/cultura/201710011072774 442-estudios-bajo-nivel-mundial/

Defensoría del Pueblo (2020). La educación frente a la emergencia sanitaria. Brechas del servicio educativo público y privado que afectan una educación a distancia accesible y de calidad. https://www.defensoria.gob.pe/wpcontent/uploads/2020/08/Serie-Informes.pdf

Gómez, A. (2017). Ideas generales sobre el método de María Montessori. Manual Práctico. ( $7^{\mathrm{a}}$ ed.). CEPE.

Gutiérrez, F. (2011). Nuevo diccionario pedagógico. La Paz, Bolivia: Gráfica Gonzáles.

Ministerio de Educación (2018). Informe de la Evaluación Censal de Estudiantes - ECE. Lima. Perú. http://umc.minedu.gob.pe/wpcontent/uploads/2019/04/presentacion-webECE2018-1.pdf

Hernández, R., Fernández, C. y Baptista, P. (2010). Metodología de la investigación. Ciudad de México: McGrawHill

Ministerio de Educación (2011). Guía metodológica para el área de Matemática. Lima, Perú: imprenta del Minedu.

Ministerio de Educación (2018). Evaluación censal de estudiantes - ECE: ¿Qué aprendizajes logran nuestros estudiantes? Amazonas. http://umc.minedu.gob.pe/wp-content/ uploads/2019/06/DRE-Amazonas-03-0519.pdf

Nunes y Bryant (2005). Las matemáticas y su aplicación: La perspectiva del niño. México: Siglo XXI.

Panizza, M. (1999). Enseñar matemática en el nivel inicial y el primer ciclo de la EGB: análisis y propuestas. Paidós.

Presidencia del Consejo de Ministros (2020). Decreto Supremo $N^{\circ}$ 044-2020-PCM, declara el estado de emergencia nacional y 
dispone el aislamiento social obligatorio, debido al Covid-19. https://www. gob .pe/institucion/pcm/normas-legales/460472044-2020-pcm

Rencoret, M. (1995). Iniciación Matemática. Andrés Bello.

Sanchidrián, C. (2017). El método de la pedagogía científica aplicada a la educación de la infancia. Biblioteca nueva.

Varas, J. y Zarco, M. (2017). Desarrollo del pensamiento matemático. https:// sites. google.com/site/matematicaycienciainfantil /matematicas/desarrollo-del-pensamientomatematico. 